Ai Wanzheng

$\mathrm{Liu} \mathrm{Hu}$

DOI: $10.21278 /$ TOF.40305

ISSN 1333-1124

eISSN 1849-1391

\title{
RESEARCH ON ENERGY DISSIPATION IN A DISCHARGE TUNNEL WITH A PLUG ENERGY DISSIPATER
}

\begin{abstract}
Summary
Plug energy dissipater, as a kind of effective energy dissipater with economic characteristics, has become very popular. The energy loss coefficient and the minimum wall pressure coefficient of the plug are two important factors in the plug energy dissipater design. In this paper, the two coefficients and relative parameters, such as the contraction ratio of the plug diameter to the flood discharge tunnel diameter, the ratio of the plug thickness to the tunnel diameter, and the Reynolds number of the flow through the plug, were analysed by theoretical considerations, and their relationship expressions were obtained by numerical simulations and were verified by experimental data. It could be concluded that the two coefficients were mainly dominated by the contraction ratio of the plug. The lower contraction ratio of the plug is, the larger are the two coefficients. The research results demonstrate that effects of the Reynolds number on the two coefficients can be neglected when this number is greater than $10^{5}$; the relative thickness of the plug has little impact on them and can be neglected.
\end{abstract}

Key words: $\quad$ Plug, contraction ratio, energy loss, wall pressure, cavitation

\section{Introduction}

With the development of hydropower projects, an increasing number of high dam hydropower projects will be carried out in future in China. The heights of some dams have to exceed and really have exceeded the level of $300 \mathrm{~m}$, such as $305 \mathrm{~m}$ and $315 \mathrm{~m}$ dams for the Jinping first-cascade hydropower project and the Shuangjiangkou hydropower project in Sichuan province, respectively [1-4]. Energy dissipation in most of the high dam projects is characterized by a deep valley, a high water head and large discharge [1-2]. Therefore, largescale diversion tunnels should be constructed during the high dam construction. In order to utilize the diversion tunnel as a permanent discharge tunnel so as to reduce investment costs, it is important to find an appropriate dissipater in the discharge tunnel to dissipate the huge amount of energy of the flow. The plug, as well as the orifice plate, as a kind of energy dissipater with sudden reduction and sudden enlargement forms, has been used in the hydropower projects because of its simple structure, convenient construction, and high energy dissipation ratio [3]. As early as 1960s, the plug energy dissipater, with the energy dissipation ratio of over $50 \%$, was used in the flood discharge tunnel of the Mica dam in 
Canada [5]. The orifice plate, similar to the plug in its energy dissipation mechanism, used in the flood discharge tunnel of the Xiaolangdi hydropower project in China, had the energy dissipation ratio of about $44 \%$ [6-7].

The flow through a plug discharge tunnel is shown in Fig. 1, where $D$ is the discharge tunnel diameter, $d$ is the plug diameter, and $T$ is the plug thickness. There exist vortex regions of ring form in the vicinity of the plug due to its sudden reduction and sudden enlargement geometry. These vortices are the original regions of energy dissipation. A number of studies [5-12] on sudden reduction and sudden enlargement of flows were focused on energy dissipation and cavitation as two main problems $\mathrm{Wu}[3,7]$ considered that the two problems were closely related to the contraction ratio of sudden reduction and sudden enlargement dissipater (contraction ratio is $d / D ; D$ and $d$ are shown in Figure 1). Tian [10-11] proved that the energy loss of either sudden flow reduction or sudden flow enlargement increased with the decrease in the contraction ratio, and he also proved that the risk of cavitation with sudden reduction and sudden enlargement dissipater is characterized by its incipient cavitation number. Liu [12] and Zhou [13] and Zhang [14] proved that the incipient cavitation number of sudden flow reduction and sudden flow enlargement decreased with the increase in the contraction ratio.

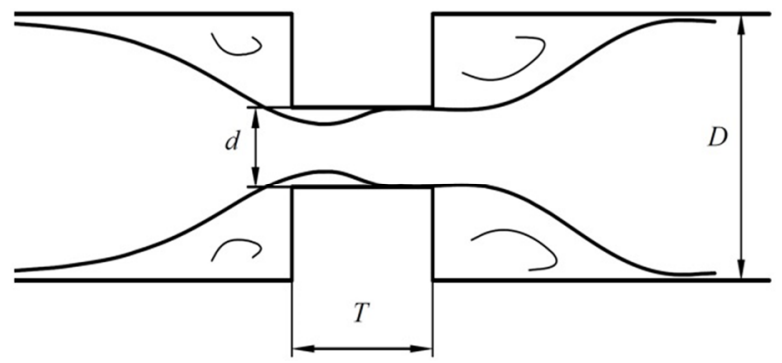

Fig. 1 Flows through a plug discharge tunnel

As stated above, the research conducted in the past focused mainly on the relationship between the contraction ratio and energy loss or incipient cavitation number [15-18]. But in actual engineering, the most practical thing is to supply the concrete expressions of plug energy loss and plug minimum wall pressure coefficients. The energy loss coefficient is related to the plug energy loss ratio. The higher is the value of the energy loss coefficient, the higher is that of the energy loss ratio. Because cavitation damage to the plug tunnel often occurs first at the position of the minimum wall pressure, the minimum wall pressure coefficient of the plug can directly reflect the cavitation characteristics of the plug discharge tunnel. With higher values of the minimum wall pressure coefficient, the risk of cavitation damage increases. Therefore, the purpose of the present study is to investigate the effects of geometric parameters, i.e., the contraction ratio and the plug thickness ( $T$ is the plug thickness in Figure 1), and of hydraulic parameters, i.e. the effects of the Reynolds number on the energy loss coefficient and on the minimum wall pressure coefficient. In addition, the aim is to present empirical expressions of energy loss coefficient and minimum wall pressure coefficient by means of numerical simulations and physical model experiments.

\section{Methodology and considerations}

The plug energy loss coefficient can be defined as follows [10-11]:

$$
\xi=\Delta p /\left(0.5 \rho u^{2}\right)
$$

where $\Delta p$ is the pressure difference between the section before $0.5 D$ plug ( $D$ is the discharge tunnel diameter), in which the flow is undisturbed, and the section after $4.0 D$ plug, where 
flows already recover normally; $\rho$ is the fluid density; $u$ is the flow average velocity in the discharge tunnel. The plug wall pressure coefficient can be defined [10-11] as

$$
C=\left(p_{\infty}-p\right) /\left(0.5 \rho u^{2}\right)
$$

where $p_{\infty}$ is the wall pressure at the undisturbed section before $0.5 D$ plug; $p$ is the wall pressure along the plug discharge tunnel. If the wall pressure $p$ reaches the lowest value $p_{\min }$, the plug wall pressure coefficient $C$ becomes the minimum wall pressure coefficient $C_{\min }$ :

$$
C_{\min }=\left(p_{\infty}-p_{\min }\right) /\left(0.5 \rho u^{2}\right)
$$

Many researchers found that the factors affecting the energy loss coefficient $\xi$ and the minimum wall pressure coefficient $C_{\min }$ were the Reynolds number, the plug relative thickness, and the contraction ratio [7, 19]. Based on that, the following expressions can be obtained [5-6]:

$$
C_{\min }, \xi=f(\beta, a, \operatorname{Re})
$$

where $R e$ is the Reynolds number; $a$ is the plug relative thickness, $a=T / D ; \beta$ is the contraction ratio, $\beta=d / D$.

\section{Numerical simulations}

\subsection{Numerical simulation model}

The commercial package FLUENT RNG $k-\varepsilon$ model was selected to calculate the hydraulic parameters of the flow through the plug because of its suitability and high precision. For steady and incompressible flows, the governing model equations include continuity, momentum, and the $k-\varepsilon$ closure.

The calculation boundary conditions are treated as follows: in the inflow boundary, the turbulent kinetic energy $k_{\text {in }}$ and the turbulent energy dissipation rate $\varepsilon_{\text {in }}$ can be defined respectively as [20]:

$$
k_{\text {in }}=0.0144 u_{\text {in }}^{2}, \varepsilon_{\text {in }}=k_{\text {in }}^{1.5} /(0.25 D)
$$

where $u_{\text {in }}$ is the average flow velocity in the inflow boundary section, which is constant $\left(u_{\text {in }}=\right.$ const $)$. In the outflow boundary, the flow is considered as fully developed. According to the continuity equation, the average flow velocity in every plug discharge tunnel section is equal. The wall boundary is controlled by the standard wall functions [2, 20, 21]. Symmetric axis boundary condition is adopted, which means that the gradient of every variable along the radial direction on the symmetry axis and the radial velocity on the symmetry axis are equal to zero $[2,20]$.

The finite volume method and a staggered grid are employed. The basic equations are integrated over each control volume to obtain discretization equations. The pressure implicit in the splitting of operator (PISO) algorithm is used to solve the velocity and pressure fields. The block-off method is adopted to treat the control volumes occupied by the plug so that the variables in these control volumes remain equal to 0 throughout the whole computation process.

\subsection{Simulation methods and phases}

A plug discharge tunnel has axial symmetry characteristics. Therefore, three dimensional numerical simulations of plug discharge tunnel flows can be reduced to two dimensional numerical simulations of the plug discharge tunnel flows. 


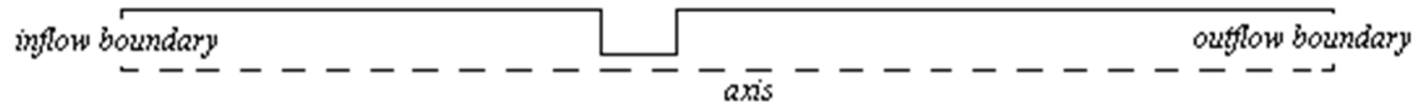

Fig. 2 The computation domain

(In the inflow boundary, $u_{\text {in }}$ is constant $\left(u_{\text {in }}=\right.$ const). In the outflow boundary, the flow is considered as fully developed. Symmetric axis boundary condition is adopted. The overall computation domain is $22.6 D$ )

The calculation diameter $(D)$ of the tunnel is $0.21 \mathrm{~m}$. The length of computational domain was selected between $10.0 \mathrm{D}$ before the plug and $12.6 \mathrm{D}$ after the plug. The computational domain is shown in Figure 2. A uniform mesh is adopted in numerical simulation. The energy loss coefficient $\xi$ is calculated by Eq. (1); the minimum wall pressure coefficient $C_{\min }$ is calculated by Eq. (3). Three kinds of calculation phases are simulated: phase No.1, to calculate the energy loss coefficient $\xi$ and the minimum wall pressure coefficient $C_{\min }$ in the range of the Reynolds number $R e=0.12 \cdot 10^{5}-27.6 \cdot 10^{5}$ when $\beta$ is 0.50 and $\alpha$ is 0.50 so as to analyse the effects of $R e$ on $\xi$ and $C_{\min }$; phase No.2, to calculate the energy loss coefficient $\xi$ at different $\beta$ and $\alpha$ when $R e$ is $1.80 \cdot 10^{5}$ so as to discuss the variations of energy loss coefficient $\xi$ with $\beta$ and $\alpha$; phase No.3, to calculate the minimum wall pressure coefficient $C_{\min }$ under the same conditions as with phase No.2 to discuss the variations of minimum wall pressure coefficient $C_{\min }$ with $\beta$ and $\alpha$.

\subsection{Results and discussions}

In order to examine the effects of grid density on simulation results, the flow field is calculated when the grid density is $10 \times 100,15 \times 150,25 \times 180,30 \times 100$ in the conditions of normal $\operatorname{Re}, \beta$ and $\alpha$. Calculation results show that the flow field tends to be stable when the grid density reaches $25 \cdot 180$ [21]. To improve the simulation accuracy, a grid of $30 \times 100$ is used and the grid self-adaptation is conducted. The values of $\mathrm{y}^{+}$are dependent on the resolution of the mesh and the Reynolds number of the flow and are defined only in the cells adjacent to the wall [21]. Simulation results show that the values of $\mathrm{y}^{+}$settle in the range 30-33, which shows that the density of the grid is satisfactory.

The distribution of wall pressure coefficient $C$ along the discharge tunnel when $\beta$ is 0.7 , $a$ is 1.0 , and $\operatorname{Re}$ is $1.80 \cdot 10^{5}$, is shown in Figure 3, where $L$ is the distance from the plug fore to some place after the plug, and $D$ is the discharge tunnel diameter. Figure 3 shows that the wall pressure coefficient $C$ reaches its maximum value at the fore of the plug, then drops gradually and becomes stable at a position far away from the $3 D$ plug.

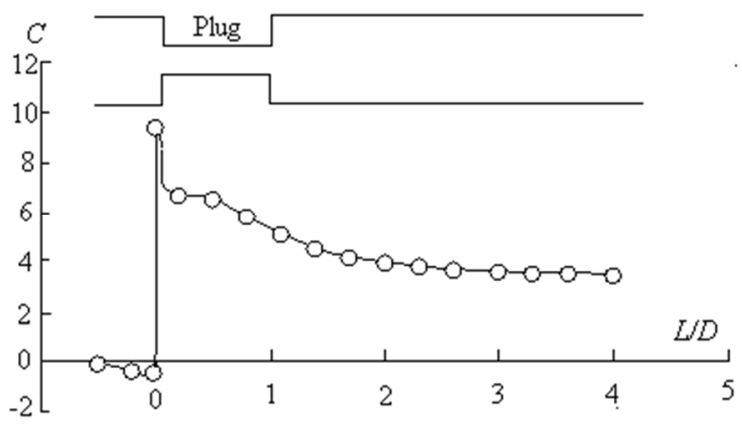

Fig. 3 Distribution of the wall pressure coefficient $C$ along the tunnel wall $(\beta=0.7, a=1.0)$

Results of phase No.1 are shown in Table 1. The data in Table 1 demonstrate that the energy loss coefficients $\xi$ and the minimum wall pressure coefficient $C_{\min }$ increase slightly with an increase in the Reynolds number $R e$ when $R e$ is less than $10^{5}$. However, when the Reynolds number $R e$ is more than $10^{5}$, these coefficients change little with the Reynolds number $R e$ change and can be considered as constants. It is can be seen that the energy loss coefficients $\xi$ and the minimum wall pressure coefficient $C_{\min }$ changed from 17.34 to 17.81 
and from 43.68 to 45.32 , respectively, with regard to the change in the Reynolds number $R e$ from $9.00 \cdot 10^{4}$ to $2.76 \cdot 10^{6}$. This phenomenon can be explained as follows: when the Reynolds number is less than $10^{5}$, the boundary layer separation is intensified with an increasing Reynolds number. As a result, the energy loss coefficient $\xi$ and the minimum wall pressure coefficient $C_{\min }$ increase slightly. But when the Reynolds number is greater than $10^{5}$, the boundary layer separation has fully developed and it is no longer affected by the Reynolds number; therefore, the energy loss coefficient $\xi$ and the minimum wall pressure coefficient $C_{\min }$ are no longer affected by the Reynolds number [2, 19, 22]. From the above analysis, it could be concluded that the effects of the Reynolds number Re on either the energy loss coefficient $\xi$ or the minimum wall pressure coefficient $C_{\min }$ can be neglected when the Reynolds number $R e$ is greater than $10^{5}$. This means that in this condition $\xi$ and $C_{\min }$ are only the functions of the plug geometric parameters.

Table 1 Variations of $\xi$ and $C_{\min }$ with $\operatorname{Re}(\beta=0.50, \alpha=0.50)$

\begin{tabular}{ccccccccc}
\hline$R e\left(\cdot 10^{5}\right)$ & 0.12 & 0.90 & 1.00 & 1.80 & 6.51 & 9.20 & 14.43 & 18.06 \\
$\xi$ & 17.34 & 17.48 & 17.81 & 17.81 & 17.81 & 17.81 & 17.81 & 17.81 \\
$C_{\min }$ & 43.68 & 44.96 & 45.32 & 45.32 & 45.32 & 45.32 & 45.32 & 45.32 \\
\hline
\end{tabular}

Figure 4 shows the distribution pattern of turbulence kinetic energy dissipation when $\operatorname{Re}$ is $2.1 \cdot 10^{5}, a$ is 0.50 , and $\beta$ is 0.50 . Generally, in the distribution pattern of turbulence kinetic energy dissipation, the turbulence kinetic energy dissipates violently in the area of intensive line. Therefore, one can conclude from Figure 4 that energy dissipates violently in the vicinity of the plug.

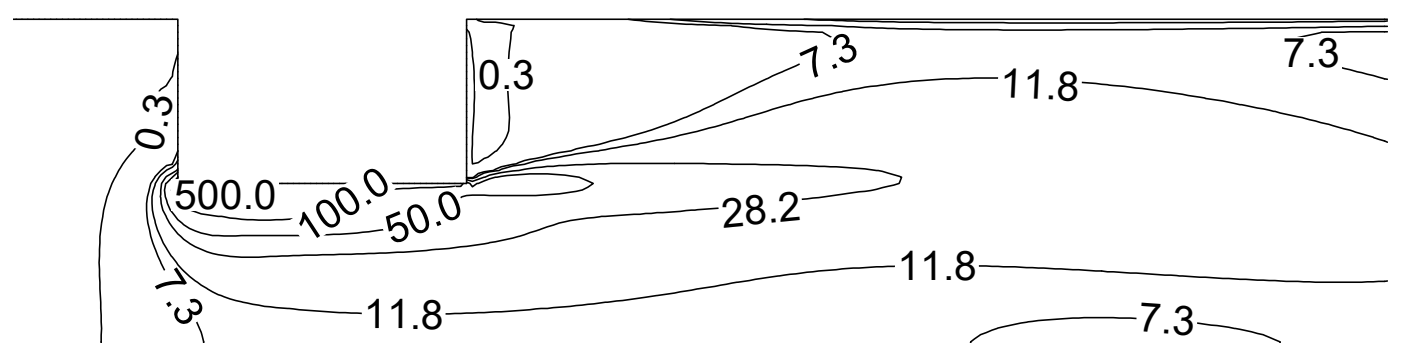

Fig. 4 Distribution of turbulence kinetic energy dissipation when $R e=2.1 \cdot 10^{5}, a=\beta=0.50$

Results of phase No.2 are shown in Table 2. The data in Table 2 show that the relative thickness $a$ has little impact on the energy loss coefficient $\xi$; therefore, the effects of relative thickness on the energy loss coefficient $\xi$ can also be neglected. It can be concluded that the energy loss coefficient $\xi$ is mainly controlled by the contraction ratio $\beta$. The graph in Figure 5 is drawn using the data from Table 2 when $a$ is 1.0. The empirical expression can be obtained by means of fitting the curve in Figure 5:

$$
\xi=0.36 \cdot \beta^{-5.7}
$$

Eq. (6) is valid for $\beta=0.4-0.8, \alpha=0.5-2.0$, and $\operatorname{Re}>10^{5}$.

Results of phase No.3 are shown in Table 3. The data in Table 3 indicate that the relative thickness $a$ hardly affects the minimum wall pressure coefficient $C_{\min }$; therefore, its effects on $C_{\min }$ can be neglected too. That means that the minimum wall pressure coefficient $C_{\min }$ is also mainly controlled by the contraction ratio $\beta$. The graph in Figure 6 is drawn using the data from Table 3 when $a$ is 1.0. The empirical expression can be obtained by means of fitting the curve in Figure 6:

$$
C_{\min }=1.894 \cdot \beta^{-4.544}
$$

Eq. (7) is valid for $\beta=0.4-0.8, \alpha=0.5-2.0$, and $R e>10^{5}$. 
Table 2 Variations of $\xi$ with $\alpha$ and $\beta\left(R e=1.80 \cdot 10^{5}\right)$

\begin{tabular}{cccccc}
\hline \multirow{2}{*}{$\alpha$} & \multicolumn{5}{c}{$\beta$} \\
\cline { 2 - 6 } & 0.4 & 0.5 & 0.6 & 0.7 & 0.8 \\
\hline 0.5 & 51.56 & 17.81 & 6.83 & 2.67 & 1.03 \\
1.0 & 50.25 & 17.59 & 6.88 & 2.62 & 1.03 \\
2.0 & 49.91 & 18.66 & 6.89 & 2.65 & 1.01 \\
\hline
\end{tabular}

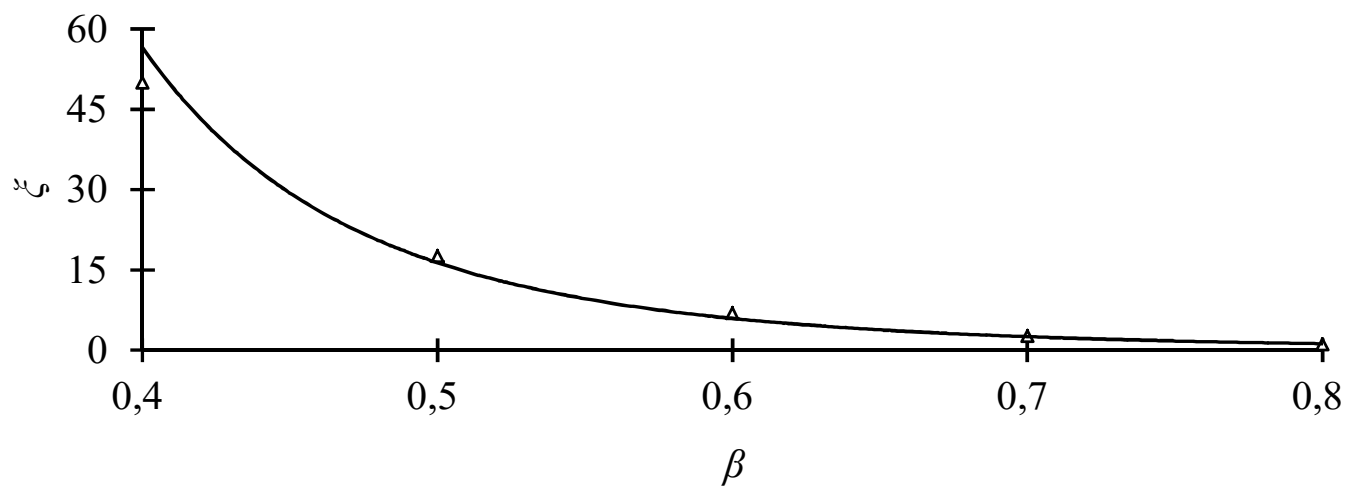

Fig. 5 Variations of $\xi$ with $\beta$ when $a=1.0\left(\operatorname{Re}=1.80 \cdot 10^{5}\right)$

Table 3 Variations of $C_{\min }$ with $\alpha$ and $\beta\left(R e=1.80 \cdot 10^{5}\right)$

\begin{tabular}{cccccc}
\hline \multirow{2}{*}{$\alpha$} & \multicolumn{5}{c}{$\beta$} \\
\cline { 2 - 6 } & 0.4 & 0.5 & 0.6 & 0.7 & 0.8 \\
\hline 0.5 & 119.54 & 45.32 & 19.23 & 9.87 & 5.13 \\
1.0 & 118.98 & 45.23 & 19.12 & 9.85 & 5.09 \\
2.0 & 118.96 & 44.98 & 19.11 & 9.84 & 5.06 \\
\hline
\end{tabular}

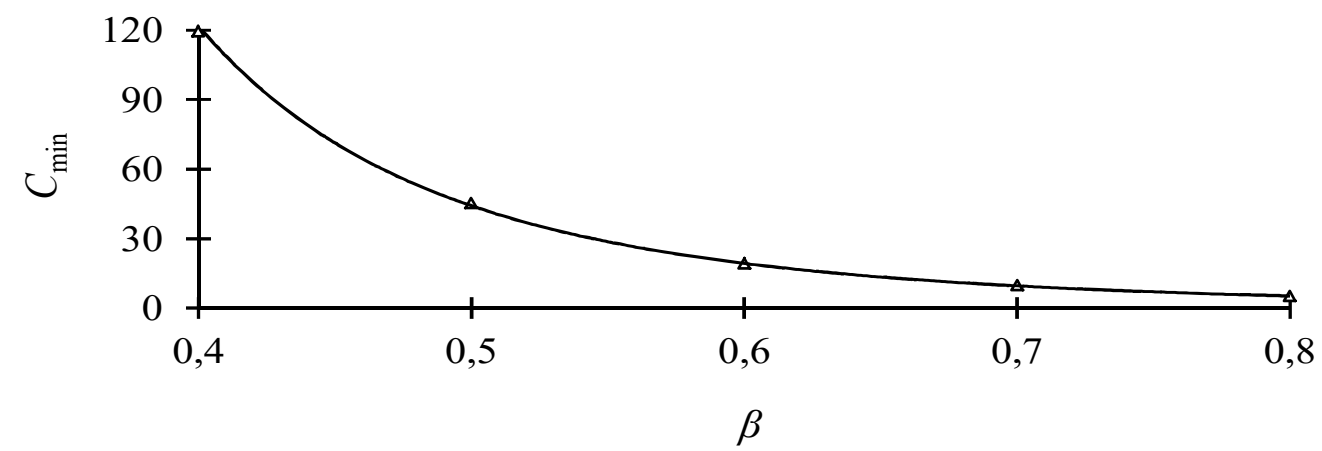

Fig. 6 Variations of $C_{\min }$ with $\beta$ when $a=1.0\left(\operatorname{Re}=1.80 \cdot 10^{5}\right)$

\section{Physical model experiments}

The physical model experimental set-up consists of an intake system, a tank, a flood discharge tunnel with a plug energy dissipater, and a return system with a rectangular weir (the physical model of the tunnel is shown in Figure 7). The diameter $(D)$ of the tunnel model is $0.21 \mathrm{~m}$, and its length is $4.75 \mathrm{~m}$, i.e., $22.6 \mathrm{D}$ from the intake to the pressure tunnel outlet at the gate. The plug energy dissipater was placed at the positions of $10.0 \mathrm{D}$ from the tunnel intake and of $12.6 \mathrm{D}$ to the outlet at the gate. The water head of about $25.0 \mathrm{D}$ can be presented 
by the intake system and the tank, which can lead to the Reynolds number Re change from $0.3 \cdot 10^{5}$ to $1.7 \cdot 10^{5}$. The opening of the gate can be changed conveniently. The geometry of the discharge tunnel in the model experiment was just the same as that in the numerical simulation. Twenty-two plastic tubes were fixed along the discharge tunnel wall and the values of the wall pressure before and after the plug were measured by measuring the water level height in the plastic tube. The precision of water level height measurement in the plastic tube is of one millimetre accuracy. The volumetric flow rate can be measured by measuring the weir and the indication of weir measurement can reach $0.000001\left(\mathrm{~m}^{3} / \mathrm{s}\right)$. The average flow velocity in the discharge tunnel can be determined by the volumetric flow rate and the cross section area of discharge tunnel. The energy loss coefficient $\xi$ was calculated using Eq. (1); the minimum wall pressure coefficient $C_{\min }$ was calculated using Eq. (3). The plug energy dissipater model is shown in Figure 8. The physical model experiments were conducted at the High-speed Flow Laboratory of Hohai University (Nanjing, China).

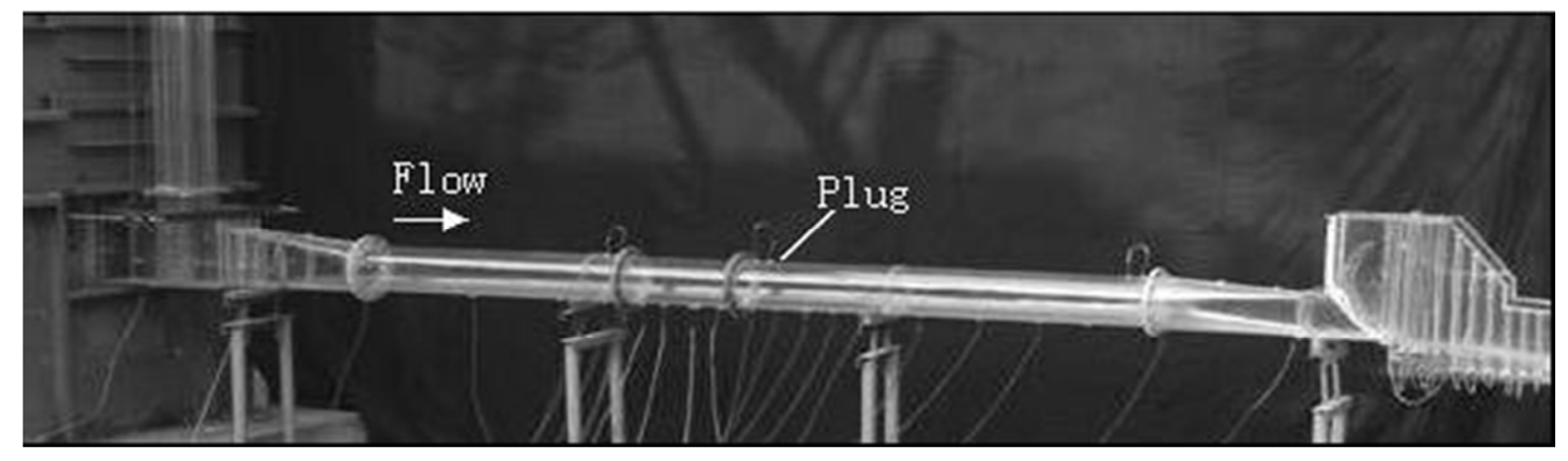

Fig. 7 Experimental model photo

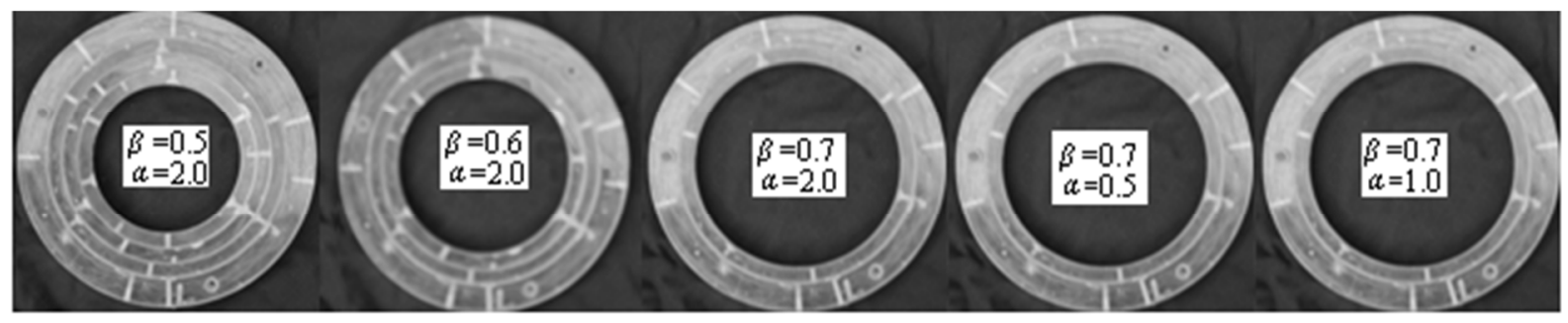

Fig. 8 Plug energy dissipater model photo

The measured energy loss coefficients $\xi$ and the minimum wall pressure coefficient $C_{\min }$ in the experiment are shown in Table 4 when $\beta$ is 0.7 and $\alpha$ is $0.5,1.0$, and 2.0. Table 4 shows that the energy loss coefficient $\xi$ and the minimum wall pressure coefficient $C_{\min }$ increase slightly with the increase in the value of the Reynolds number $R e$ when $R e$ is less than $10^{5}$, but when the Reynolds number $R e$ is greater than $10^{5}$, the Reynolds number $R e$ has a minimal effect on the two coefficients. The relative thickness $a$ has a minimal impact on both the energy loss coefficient $\xi$ and the minimum wall pressure coefficient $C_{\min }$. The experiment conclusions stated above were in agreement with those of numerical simulation, which proves that it is possible to investigate plug discharge tunnel flows by using the FLUENT RNG $k-\varepsilon$ model. 
Table 4 Experiment results when $\beta$ was 0.7 and $R e$ and $\alpha$ varied

\begin{tabular}{ccccc}
\hline \multirow{2}{*}{$\alpha$} & \multicolumn{4}{c}{$\operatorname{Re}\left(\cdot 10^{5}\right)$} \\
\cline { 2 - 5 } & 1.5 & 1.3 & 1.0 & 0.4 \\
\hline \multirow{2}{*}{0.5} & $\xi=2.763$, & $\xi=2.762$, & $\xi=2.765$, & $\xi=2.733$, \\
& $C_{\min }=9.513$ & $C_{\min }=9.515$ & $C_{\min }=9.513$ & $C_{\min }=9.488$ \\
\multirow{2}{*}{1.0} & $\xi=2.761$, & $\xi=2.760$, & $\xi=2.762$, & $\xi=2.721$, \\
& $C_{\min }=9.511$ & $C_{\min }=9.501$ & $C_{\min }=9.514$ & $C_{\min }=9.378$ \\
\multirow{2}{*}{2.0} & $\xi=2.751$, & $\xi=2.750$, & $\xi=2.753$, & $\xi=2.719$, \\
& $C_{\min }=9.413$ & $C_{\min }=9.403$ & $C_{\min }=9.415$ & $C_{\min }=9.343$ \\
\hline
\end{tabular}

The measured energy loss coefficient $\xi$ and the minimum wall pressure coefficient $C_{\min }$ when $\alpha$ was 2.0 and $R e$ was $1.3 \cdot 10^{5}$ are shown in Table 5, together with the results of comparison between the model experimental data and the data calculated by using Eq.(6) and Eq.(7). In Table 5, $\mathrm{E}_{\mathrm{r} 1}$ and $\mathrm{E}_{\mathrm{r} 2}$ are defined as follows:

$$
\begin{aligned}
& E_{r 1}=\frac{\left|\xi_{c a l}-\xi_{m}\right|}{\xi_{c a l}} \cdot 100 \% \\
& E_{r 2}=\frac{\left|C_{\text {min.cal }}-C_{\text {min.m }}\right|}{C_{\text {min.cal }}} \cdot 100 \%
\end{aligned}
$$

where $\xi_{\text {cal }}$ is the energy loss coefficient calculated by using Eq.(6); $\xi_{\mathrm{m}}$ is the energy loss coefficient obtained by the model experiment; $C_{\text {min.cal }}$ is the minimum wall pressure coefficient calculated by using Eq.(7); $C_{\text {min.m }}$ is the minimum wall pressure coefficient obtained by the model experiment. $\mathrm{E}_{\mathrm{r} 1}$ demonstrates the deviation of the model experiment energy loss coefficient from the energy loss coefficient calculated by means of Eq.(6), and $\mathrm{E}_{\mathrm{r} 2}$ demonstrates the difference between the model experiment minimum wall pressure coefficient and the minimum wall pressure coefficient calculated by means of Eq.(7). The results in Table 5 show that the average error of Eq. (6) is $2.52 \%$, the average error of Eq. (7) is $1.53 \%$, while the maximum error of Eq. (6) is less than $7.5 \%$ and the maximum error of Eq. (7) is less than $1.8 \%$. The comparison results show that it is effective to calculate the energy loss coefficient $\xi$ of the plug energy dissipater by means of Eq. (6) and to calculate the minimum wall pressure coefficient $C_{\min }$ of the plug energy dissipater by means of Eq.(7).

Table 5 Comparison between experiment results and calculation results $\left(\alpha=2.0\right.$ and $\left.R e=1.3 \cdot 10^{5}\right)$

\begin{tabular}{ccccccc}
\hline$\beta$ & $\xi_{\mathrm{m}}$ & $\xi_{\text {cal }}$ & $C_{\text {min.m }}$ & $C_{\text {min.cal }}$ & $E_{\mathrm{r} 1}(\%)$ & $E_{\mathrm{r} 2}(\%)$ \\
0.5 & 17.33 & 18.72 & 44.98 & 44.18 & 7.41 & 1.80 \\
0.6 & 6.61 & 6.62 & 19.50 & 19.30 & 0.15 & 1.06 \\
0.7 & 2.75 & 2.75 & 9.41 & 9.58 & 0.02 & 1.75 \\
\hline
\end{tabular}

\section{Conclusions}

For the plug energy dissipater, the effects of the Reynolds number $R e$ on its energy loss coefficient $\xi$ and on its minimum wall pressure coefficient $C_{\min }$ can be neglected when this number is greater than $10^{5}$. The contraction ratio $\beta$ is the key factor that affects the energy loss coefficient $\xi$ as well as the minimum wall pressure coefficient $C_{\min }$. The lower is the contraction ratio $\beta$, the greater are the values of the energy loss coefficient $\xi$ and the minimum wall pressure coefficient $C_{\min }$. The relationship between $\xi$ and $\beta$ can be expressed by Eq. (6), and the relationship between $C_{\min }$ and $\beta$ can be expressed by Eq. (7). Compared with experimental values, the maximum error of Eq. (6) is less than $7.5 \%$, the maximum error of Eq. (7) is less than 1.8\%. Equations (6) and (7) were proved by experiment data. 


\section{Funding}

Project supported by the Open Foundation from Marine Sciences in the Most Important Subjects of Zhejiang (No. 20160203).

\section{REFERENCES}

[1] Zhou J, Yang Z, Chen G. Status and challenges of high dam in China. J Hydraulic Engineering 2006; 37:1433-1438 (in Chinese)

[2] Xia Q, Ni H. (2003). Numerical simulation of plug energy dissipator. J Hydraulic Engineering. 2003; 8: 37-42 (in Chinese).

[3] Wu J, Chai G, Xiang T. Hydraulic characteristics and optimization of orifice plate discharge tunnel of the Xiaolangdi hydropower project. J Hydraulic Engineering, Sup 1995; 101-109 (in Chinese)

[4] Ai W, Zhou Q. Hydraulic characteristics of multi-stage orifice plate. J Shanghai Jiaotong University (science) 2014; 19:361-366. DOI: 10.1007/s12204-014-1510-X

[5] Russell So and Ball JW. Sudden-enlargement energy dissipater for Mica dam. J Hydr Eng Div: ASCE 1967; 93: 41-56.

[6] Zhang Z, Cai J. Compromise orifice geometry to minimize pressure drop. J Hydraulic Engineering 1999; 11: 1150-1153. DOI: 10.1061/(ASCE)0733-9429(1999)125:11(1150)

[7] Wu J, Ai W. Head loss coefficient of orifice plate energy dissipaters. J Hydraulic Research 2010; 48:526530. DOI: $10.1080 / 00221686.2010 .507347$

[8] Ai W, Wu J. Comparison on hydraulic characteristics between orifice plate and plug. J Shanghai Jiaotong University(science) 2014;19: 476-480. DOI: 10.1007/s12204-014-1527-1

[9] Xia Q, Ni H. Numerical simulation of plug energy dissipater. J Hydraulic Engineering 2003; 8: 37-42 (in Chinese)

[10] Tian Z, Xu W, Wang W, Liu S. Hydraulic characteristics of plug energy dissipater in flood discharge tunnel. J Hydrodynamics, Ser. B 2009; 21:799-806.

[11] Tian Z, Xu W, Liu S. Numerical simulation of composite plug energy dissipater. J Advances in Science and Technology of Water Resources 2005;25: 8-10 (in Chinese)

[12] Liu S, Yang Y, Xu W, Wang W. Hydraulic characteristics of throat-type energy dissipater in discharge tunnels. J Hydraulic Engineering 2002; 7: $42-50$ (in Chinese)

[13] Zhou H. Numerical analysis of the 3-D flow field of pressure atomizers with $\mathrm{V}$-shaped cut at orifice. J Hydrodynamics, Ser.B 2011; 23: 187-192.

[14] Zhang C, Yang Y. 3-D numerical simulation of flow through an orifice spill way tunnel. J Hydrodynamics, Ser. B 2002; 3:83-90.

[15] Li H, Chen H. Formation and influencing factors of free surface vortex in a barrel with a central orifice at bottom. J Hydrodynamics, Ser. B 2009;21:238-244.

[16] Cai G, Wu J, Wang H. Cavitation and energy dissipation of the bottom tunnel with orifice plates in the Xiaolangdi dam. Proceedings of International Symposium on Cavitation and Erosion in Hydraulic Structures and Machinery, Nanjing of China 1992: 83-90.

[17] Fossa, M, Guglielmini G. Pressure drop and void fraction profiles during horizontal flow through thin and thick orifices. J Experimental Thermal and Fluid Science 2002; 26: 513-523. DOI: 10.1016/S08941777(02)00156-5

[18] Ai W, Ding T. Orifice plate cavitation mechanism and its influencing factors. J Water Science and Engineering 2010; 3: 321-330.

[19] Ai W, Wang J. Minimum wall pressure coefficient of orifice plate energy dissipater. J Water Science and Engineering 2015; 8: 85-88. DOI: 10.1016/j.wse.2014.06.001

[20] Wu J, Ai W. Flows through energy dissipaters with sudden reduction and sudden enlargement forms. J Hydrodynamics, Ser.B 2010; 22:234-343.

[21] Zhang C, Yang Y. 3-D numerical simulation of flow through an orifice spill-way tunnel. J Hydrodynamics, Ser.B 2002; 3:83-90.

[22] Tian Z. Study on the Hydraulic Characteristics of Plug Energy Dissipater. D Dr. paper of Sichuan University of China 2006: 36-50. 


\section{Appendix I}

\section{Notation}

$d \quad$ plug diameter.

D flood discharge tunnel diameter.

$T \quad$ thickness of the plug.

$g \quad$ acceleration of gravity.

$u \quad$ section average velocity in the flood discharge tunnel.

$\rho \quad$ density of water.

$\Delta p \quad$ pressure difference between the section before $0.5 \mathrm{D}$ plug and the section after $4.0 \mathrm{D}$ plug.

$\xi \quad$ energy loss coefficient of the plug.

a $\quad T / D$, relative thickness of the plug.

$\beta \quad d / D$, contraction ratio.

$\mu \quad$ dynamic viscosity of water.

Re $\quad u D /(\mu / \rho)$, the Reynolds number of flow in the flood discharge tunnel.

$p \quad$ discharge tunnel wall pressure.

$C \quad$ wall pressure coefficient of the plug.

$C_{\text {min }} \quad$ minimum wall pressure coefficient around the plug.

$p_{\infty} \quad$ wall pressure at the undisturbed section before $0.5 \mathrm{D}$ plug.

$p_{\min } \quad$ lowest wall pressure around the plug.

$k_{\text {in }} \quad$ turbulent kinetic energy in the inflow boundary.

$\varepsilon_{\text {in }} \quad$ turbulent energy dissipation rate in the inflow boundary.

$u_{\text {in }} \quad$ average velocity in the inflow boundary.

$\xi_{\text {cal }} \quad$ energy loss coefficient calculated by using Eq.(7).

$\xi_{\mathrm{m}} \quad$ energy loss coefficient obtained by model experiment.

$C_{\text {min.cal }}$ minimum wall pressure coefficient calculated by using Eq.(8).

$C_{\text {min.m }} \quad$ minimum wall pressure coefficient obtained by model experiment.

Submitted: $\quad 13.01 .2016$

Accepted: $\quad 20.9 .2016$
Ai Wanzheng

Liu $\mathrm{Hu}$, corresponding author mailto:liuhu-175@163.com

Marine College of Zhejiang Ocean

University, Zhoushan 316000, P. R. China 